\title{
Expression of miR-21, miR-31, miR-96 and miR-135b is correlated with the clinical parameters of colorectal cancer
}

\author{
XIN MIN XU ${ }^{1,2,6^{*}}$, JIAN CHANG QIAN ${ }^{3,6^{*}}, \mathrm{ZHOU} \mathrm{LU} \mathrm{DENG}^{4}, \mathrm{ZHE} \mathrm{CAI}^{5}$, \\ TAO TANG ${ }^{4}$, PENG WANG $^{4}, \mathrm{KE} \mathrm{HUA} \mathrm{ZHANG}^{5}$ and JIAN-PING CAI $^{6}$ \\ ${ }^{1}$ Graduate School, Chinese Academy of Medical Sciences and Peking Union Medical College, Beijing 100730; \\ ${ }^{2}$ National Center for Clinical Laboratories, Beijing 100730; ${ }^{3}$ Department of Pharmacology, \\ Wenzhou Medical College, Zhejiang 325035; Departments of ${ }^{4}$ General Surgery and ${ }^{5}$ Immunology, \\ Institute of Clinical Medicine, China-Japan Friendship Hospital, Beijing 100029; ${ }^{6}$ The Key Laboratory of Geriatrics, \\ Beijing Hospital and Beijing Institute of Geriatrics, Ministry of Health, Beijing 100730, P.R. China
}

Received February 13, 2012; Accepted May 1, 2012

DOI: $10.3892 / \mathrm{ol} .2012 .714$

\begin{abstract}
The aim of this study was to determine the expression of miR-21, miR-31, miR-96 and miR-135b in 52 paired colorectal cancer (CRC) tissues and to analyze the correlation between microRNAs (miRNAs) and clinicopathological features. We developed a quantification method that relies on a standard plot, constructed from known concentrations of standards, in order to measure the number of miRNAs. In addition to this, we analyzed the expression levels of miR-21, miR-31, miR-96 and miR-135b in 52 cases of primary CRC and corresponding normal mucosal tissue using real-time PCR with SYBR-Green I. An independent sample t-test was used to compare the differential expression between tumor tissues and normal mucosal tissues. The Mann-Whitney U and Kruskall-Wallis tests were used to compare the correlation between miRNA expression levels and clinicopathological features. The expression of miR-21, miR-31, miR-96 and miR-135b was upregulated in the CRC tissues compared to normal mucosal tissues $(\mathrm{P}<0.05)$. Furthermore, miR-21 and miR-135b were positively correlated with the clinical stage $(\mathrm{P}=0.048$ and $\mathrm{P}=0.029$, respectively), while miR-96 and miR-135b were correlated with liver metastasis $(P=0.006$ and $\mathrm{P}=0.013$, respectively). Our results suggest that miR-21, miR-31, miR-96 and miR-135b may function in the process of CRC development and progression. miR-135b levels in particular may correlate with the degree of malignancy.
\end{abstract}

Correspondence to: Dr Jian-Ping Cai, The Key Laboratory of Geriatrics, Beijing Hospital and Beijing Institute of Geriatrics, Ministry of Health, No. 1 DaHua Road, Dong Dan, Beijing 100730, P.R. China

E-mail: caijp61@vip.sina.com

*Contributed equally

Key words: microRNA, colorectal cancer, real-time RT-PCR

\section{Introduction}

Colorectal cancer (CRC) is the third most common type of malignant neoplasm and the third leading cause of cancer-related mortalities worldwide (1). Conventional pathological diagnosis is traumatic, with the majority of cases being detected in the later stage. Early detection of CRC has puzzled clinicians and scientists for years. Even with annual fecal occult-blood testing, which has decreased the 13-year cumulative mortality rate from CRC by $33 \%$ (2), the outcome remains poor in patients with advanced disease. Only CRC diagnosed at an early stage is likely to be cured by surgical resection. Genetic alterations present in CRC, including those in APC, $\mathrm{K}$-ras or p53, do not demonstrate a confirmed correlation between their mutation rate and clinical stage (3). Therefore, a reliable, sensitive and specific molecular diagnostic test for $\mathrm{CRC}$ is highly desirable from a clinical perspective.

Accumulating evidence suggests that microRNAs (miRNAs) play a crucial role in the tumorigenesis and prognosis of cancer (4-7). miRNAs are non-coding, single-stranded RNAs of 18-25 nucleotides in length, which are able to regulate gene expression by inhibiting translation or decreasing stability of target mRNAs. Over the past few years, interest in the identification, detection and utilization of miRNA molecules has expanded rapidly. Bioinformatic analysis suggests that up to $30 \%$ of human genes may be regulated by miRNAs, despite the fact that they only constitute approximately $1 \%$ of the human genome $(8,9)$.

Changes in miRNA expression have been observed in a variety of human tumors, including breast (5), prostate (6), hepatocellular (7), colorectal (10) and oral cancer (11), chronic lymphocytic leukemia (12) and bladder cancer (13) (Table I). miRNAs are involved oncogenesis, disease progression, invasion and metastasis, and are associated with patient prognosis $(4,14)$. Studies have confirmed that the expression of miR-21 and miR-31 is upregulated in CRC patients (15). miR-21 has been demonstrated to accelerate tumorigenesis by targeting tumor suppressor genes, including phosphatase and tensin homolog (PTEN), tropomyosin 1 (TPM1) and 
programmed cell death 4 (PDCD4) $(7,16,17)$. Ultimately, this increases tumor cell growth, migration and invasion.

Recent studies have demonstrated that miR-21 and miR-31 are positive regulators of colon carcinoma cells with migratory and invasive properties. As the direct target gene of miR-21 and miR-31, the T lymphoma and metastasis gene 1 (TIAM1) has been found to regulate the migration and invasion of colon carcinoma cells (18). Bandrés et al (15) examined the expression of 156 mature miRNAs using real-time PCR in 15 CRC cell lines as well as 12 matched pairs of tumoral and nontumoral adjacent tissues. These authors found that miR-31, miR-96 and miR-135b were upregulated in both cell lines and tissues and the expression level of miR-31 was correlated with the clinical stage of CRC. Another study confirmed that there was a considerable upregulation of miR-135b in CRC (19). The same study also revealed that the two miR-135, isoforms $a$ and $b$, correlated with low APC mRNA levels, suggesting that miR-135a and miR-135b contribute to the pathogenesis of CRC.

Accordingly, we predicted that miR-21, miR-31, miR-96 and miR-135b may function as micro-oncogenes in colorectal carcinogenesis and regulate CRC development and progression.

In the present study, we developed a highly sensitive and specific quantitative real-time PCR (qPCR) method based on SYBR-Green I. This was used to detect the expression of miR-21, miR-31, miR-96 and miR-135b and to analyze their correlation to the clinicopathological parameters of CRC. An understanding of the miRNA association with CRC clinicopathological features is essential to gain an insight into the miRNA involvement in colorectal carcinogenesis. This method is less costly than the method based on the TaqMan probe assay. Therefore, it may become more widely utilized in conventional laboratories, as well as used for the analysis of numerous samples.

\section{Materials and methods}

Study population and tissue sample preparation. In the present study, we recruited 52 patients with CRC, who were diagnosed and received surgery at the China-Japan Friendship Hospital, Beijing, China, between October 2009 and December 2010. After obtaining written informed consent from all subjects or their guardians and approval from the Beijing Hospital Institutional Review Committee, resected tumors and the corresponding adjacent normal mucosa tissues were obtained from surgically treated patients with CRC. Samples were immediately stored in liquid nitrogen until RNA extraction was performed. Generally, normal mucosal tissue was obtained from a region $>10 \mathrm{~cm}$ away from the cancer tissue. The eligible tumors had to result from primary CRC without prior preoperative radiotherapy or chemotherapy treatment. The study group comprised 26 males and 26 females, with a median age of 62 years, range 36-89. The TNM classification was in accordance with the American Joint Committee on Cancer (AJCC) TNM staging. Patient details, including gender, tumor location, TNM stage, grade and lymphovascular invasion, are shown in Table II.

Total RNAs of the tumor tissues and the corresponding normal tissues were isolated using the mirVana miRNA Isolation kit (Ambion, Carlsbad, CA, USA), according to the manufacturer's instructions. RNA concentration and purity were analyzed using a UV spectrophotometer (A260/A280 $>2.0 ;$ A260/A230 >1.8).

Preparation and quantification of standards. Reliable standards are essential in qPCR analysis. Therefore, we selected a cloned circular plasmid, which is more stable than PCR products (20). The four miRNAs (miR-21, miR-31, miR-96 and miR-135b) and U6 snRNA from the HT29 cells were reversetranscribed. The target miRNAs were then amplified according to the procedures described below. Following these procedures, the products were cloned into a pMD-18T vector (Takara, Otsu, Shiga, Japan), the ligated fragments were transformed into DH5 $\alpha$ competent cells and the transformed cultures were spread onto lysogeny broth plates containing ampicillin $(75 \mu \mathrm{g} / \mathrm{ml})$. Clones were screened by PCR reactions and positive clones were selected and processed for plasmid isolation. The purity and concentration of the plasmids were accurately quantified using a Qubit ${ }^{\mathrm{TM}}$ dsDNA BR Assay kit (Invitrogen, Ltd., Inchinnan Business Park, UK). The exact sequence of the inserted plasmids was analyzed and confirmed by sequencing with RV-M universal primers. We obtained a copy number based upon the molecular weight of the plasmid and insert (21). The plasmids were then diluted in $1 \mathrm{X}$ Tris-EDTA (TE) to $10^{10} \mathrm{copy} / \mu \mathrm{l}$. To maximize accuracy, dilutions were performed over a range of copy numbers that included the amount of target mRNA expected in the experimental RNA samples. Thus, serial 10 -fold dilutions from $10^{10}$ to $10^{0} \mathrm{copy} / \mu \mathrm{l}$ of the plasmids were used as standards. Plots of the logarithm of the template concentration versus the $\mathrm{Ct}$ were graphed, and the PCR efficiency was calculated using the equation: $\mathrm{E}=10(-1 / \mathrm{slope})(22)$.

Reverse transcription and real-time PCR. Stem-loop real-time RT-PCR was used to analyze the expression of miRNA. We designed miR-21, miR-31, miR-96 and miR-135b stem-loop RT primers and amplification primers according to the method developed by Chen et al (23). cDNAs were synthesized from the total RNA using unique stem-loop RT primers. The sequences were as follows: 5'-GTCGTATCCAGTGCAGG GTCCGAGGTATTCGCACTGGATACGACTCAACA-3' (miR-21), 5'-GTCGTATCCAGTGCAGGGTCCGAGGT ATTCGCACTGGATACGACAGCTAT-3' (miR-31), 5'-GTCGTATCCAGTGCAGGGTCCGAGGTATTCGCACT GGATACGACAGCAAA-3' (miR-96) and 5'-GTCGT ATCCAGTGCAGGGTCCGAGGTATTCGCACTGGATAC GACTCACAT-3' (miR-135b). Reverse transcriptase reactions contained the following reagents: $10 \mathrm{ng}$ RNA sample, $60 \mathrm{nM}$ stem-loop RT primer, 1X RT buffer, $0.25 \mathrm{mM}$ each of dNTP, $4 \mathrm{U} / \mu 1 \mathrm{M}-\mathrm{MLV}$ reverse transcriptase (Promega, Madison, WI, USA) and $0.4 \mathrm{U} / \mu \mathrm{l}$ RNase inhibitor (Takara). Reactions (10 $\mu \mathrm{l})$ were incubated in a GenAmp PCR System 9700 (Applied Biosystems, Foster City, CA, USA) at $16^{\circ} \mathrm{C}$ for $30 \mathrm{~min}, 42^{\circ} \mathrm{C}$ for $30 \mathrm{~min}$ and $85^{\circ} \mathrm{C}$ for $5 \mathrm{~min}$. The samples were then held at $4^{\circ} \mathrm{C}$. Real-time PCR was performed using the Thermal Cycler Dice real-time system TP800 (Takara). The universal reverse primer for the four miRNAs was 5'-CAGTGCAGGGTCC GAGGT-3'. The specific forward primers were as follows: 5'-GCCCGCTAGCTTATCAGACTGATG-3' (miR-21), 5'-GCCGCAGGCAAGATGCTGGC-3' (miR-31), 5'-GCCC GCTTTGGCACTAGCACATT-3' (miR-96) and 5'-GCCCG 
Table I. Mean fold change of analyzed miRNAs in colorectal tumor samples.

\begin{tabular}{|c|c|c|c|c|}
\hline miRNA & $\begin{array}{l}\text { Mean fold } \\
\text { change }\end{array}$ & $\begin{array}{l}\text { Chromosome } \\
\text { localization }\end{array}$ & $\begin{array}{l}\text { Correlation with cancer } \\
\text { in previous studies (Refs.) }\end{array}$ & Putative targets (Refs.) \\
\hline miR-21 & 1.5 & $17 \mathrm{q} 23.2$ & $\begin{array}{l}\uparrow \mathrm{CRC}(10,15), \uparrow \mathrm{CLL}(12), \uparrow \text { breast cancer }(5), \\
\uparrow \text { pancreatic cancer }(32), \uparrow \mathrm{HCC}(7)\end{array}$ & $\begin{array}{l}\text { PTEN }(5,7), \text { TPM1 (16), } \\
\text { PDCD4 (17), TIAM1 (18) }\end{array}$ \\
\hline $\operatorname{miR}-31$ & 33.5 & $9 p 21.3$ & $\uparrow \mathrm{CRC}(10,15,29), \uparrow$ oral cancer $(11)$ & $\begin{array}{l}\text { FOXC-2, FOXC-3 (15), } \\
\text { SATB2 (24), TIAM1 (18) }\end{array}$ \\
\hline miR-96 & 2.2 & $7 q 32.2$ & $\begin{array}{l}\uparrow \mathrm{CRC}(15), \uparrow \text { bladder cancer }(13),(26) \\
\uparrow \text { prostate carcinoma }(6), \downarrow \text { pancreatic cancer }(26)\end{array}$ & K-ras (26), CHES1 (15) \\
\hline miR-135b & 17.9 & $1 \mathrm{q} 32.1$ & $\uparrow$ CRC $(15,19), \uparrow$ prostate cancer $(33)$ & MSH2 (15), APC (19) \\
\hline
\end{tabular}

CRC, colorectal cancer; CLL, chronic lymphocytic leukemia; HCC, human hepatocellular cancer.

Table II. Correlation between miR-21, miR-31, miR-96 and miR-135b relative expression levels and clinicopathological features in CRC patients.

\begin{tabular}{|c|c|c|c|c|c|c|c|c|c|}
\hline & \multirow[b]{2}{*}{$\mathrm{n}$} & \multicolumn{2}{|c|}{ miR-21 } & \multicolumn{2}{|c|}{$\operatorname{miR}-31^{\mathrm{a}}$} & \multicolumn{2}{|c|}{$\operatorname{miR}-96^{\mathrm{b}}$} & \multicolumn{2}{|c|}{$\operatorname{miR}-135 b^{a}$} \\
\hline & & Mean \pm SD & P-value & Mean \pm SD & P-value & Mean \pm SD & $\mathrm{P}$-value & Mean $\pm \mathrm{SD}$ & P-value \\
\hline \multicolumn{10}{|l|}{ Tissue } \\
\hline Cancer & 52 & $4.803 \pm 3.037$ & 0.003 & $0.804 \pm 0.851$ & 0.003 & $1.242 \pm 0.961$ & 0.013 & $1.167 \pm 1.186$ & $<0.001$ \\
\hline Normal & 52 & $3.192 \pm 2.242$ & & $0.024 \pm 0.030$ & & $0.553 \pm 0.811$ & & $0.065 \pm 0.074$ & \\
\hline \multicolumn{10}{|l|}{ Gender } \\
\hline Male & 26 & $4.603 \pm 3.150$ & 0.4 & $0.644 \pm 0.846$ & 0.528 & $1.153 \pm 0.857$ & 0.286 & $1.161 \pm 1.153$ & 0.808 \\
\hline Female & 26 & $5.003 \pm 2.969$ & & $0.399 \pm 0.628$ & & $1.334 \pm 1.069$ & & $1.239 \pm 1.510$ & \\
\hline \multicolumn{10}{|l|}{ Localization } \\
\hline Right side & 17 & $4.330 \pm 3.344$ & 0.067 & $0.852 \pm 1.093$ & 0.493 & $1.119 \pm 0.990$ & 0.628 & $0.883 \pm 0.849$ & 0.2 \\
\hline Left side & 35 & $5.033 \pm 2.900$ & & $0.448 \pm 0.671$ & & $1.625 \pm 2.126$ & & $1.486 \pm 1.661$ & \\
\hline \multicolumn{10}{|l|}{ Lymph node } \\
\hline Positive & 32 & $6.560 \pm 2.805$ & 0.032 & $0.498 \pm 0.757$ & 0.529 & $1.358 \pm 1.039$ & 0.829 & $1.319 \pm 1.332$ & 0.127 \\
\hline Negative & 20 & $4.191 \pm 3.416$ & & $0.714 \pm 0.976$ & & $1.062 \pm 0.819$ & & $0.932 \pm 0.898$ & \\
\hline \multicolumn{10}{|c|}{ Differentiation } \\
\hline High & 11 & $5.925 \pm 4.546$ & 0.348 & $0.816 \pm 1.198$ & 0.968 & $0.975 \pm 0.828$ & 0.12 & $1.058 \pm 1.194$ & 0.194 \\
\hline Moderate & 31 & $4.847 \pm 2.634$ & & $0.427 \pm 0.593$ & & $1.452 \pm 0.995$ & & $1.353 \pm 1.261$ & \\
\hline Low & 10 & $3.433 \pm 1.600$ & & $0.414 \pm 0.494$ & & $0.855 \pm 0.865$ & & $0.702 \pm 0.849$ & \\
\hline \multicolumn{10}{|l|}{ Stage } \\
\hline II & 19 & $3.782 \pm 1.460$ & 0.048 & $0.462 \pm 0.454$ & 0.76 & $1.055 \pm 0.751$ & 0.063 & $1.206 \pm 1.098$ & 0.029 \\
\hline III & 24 & $4.272 \pm 2.120$ & & $0.697 \pm 0.970$ & & $1.156 \pm 0.932$ & & $1.002 \pm 1.508$ & \\
\hline IV & 6 & $5.828 \pm 2.948$ & & $0.145 \pm 0.107$ & & $2.298 \pm 1.283$ & & $2.826 \pm 1.903$ & \\
\hline \multicolumn{10}{|c|}{ Liver metastasis } \\
\hline Yes & 7 & $5.076 \pm 3.113$ & 0.778 & $0.131 \pm 0.104$ & 0.29 & $2.387 \pm 1.195$ & 0.006 & $2.560 \pm 1.875$ & 0.013 \\
\hline No & 45 & $4.760 \pm 3.059$ & & $0.655 \pm 0.890$ & & $1.060 \pm 0.792$ & & $0.946 \pm 0.883$ & \\
\hline
\end{tabular}

Significant differences $(\mathrm{P}<0.05)$ are noted in bold. ${ }^{a}$ Median of relative expression $x 10 .{ }^{b}$ Median of relative expression x100. CRC, colorectal cancer.

CTATGGCTTTCATTCCT-3' (miR-135b). The $25 \mu$ PCR reaction mixture included $1 X \mathrm{SYBR}$ premix Ex Taq mix (Takara), $2 \mu \mathrm{l}$ RT products and $10 \mathrm{nM}$ of each forward and reverse primer. Reactions were incubated in a 96 -well plate at $95^{\circ} \mathrm{C}$ for $30 \mathrm{sec}$, followed by 45 cycles of $95^{\circ} \mathrm{C}$ for $15 \mathrm{sec}$ and $60^{\circ} \mathrm{C}$ for $21 \mathrm{sec}$. Dissociation from 65 to $95^{\circ} \mathrm{C}$ was conducted to confirm the specificity of the amplification products. The threshold cycle data were determined using second derivative max settings. U6 was used as an internal control to normalize the level of target miRNAs. The stem-loop RT primers and amplification primers of U6 were obtained from Ribobio Co., Ltd., Guangzhou, China). 
A

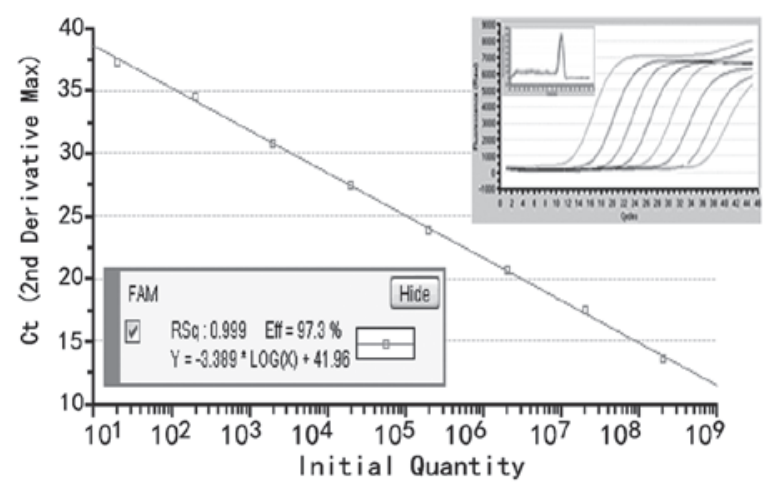

C

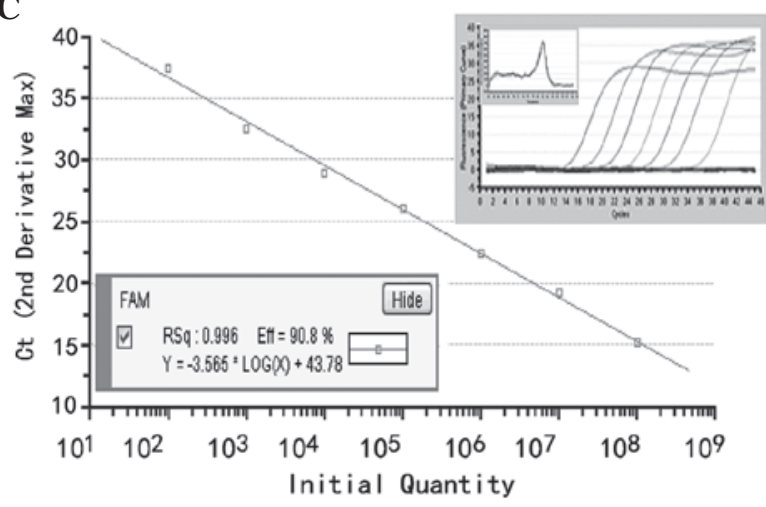

B

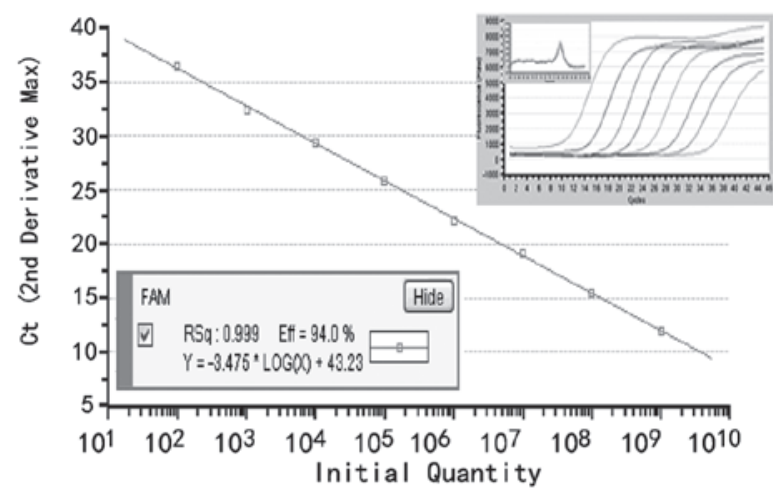

D

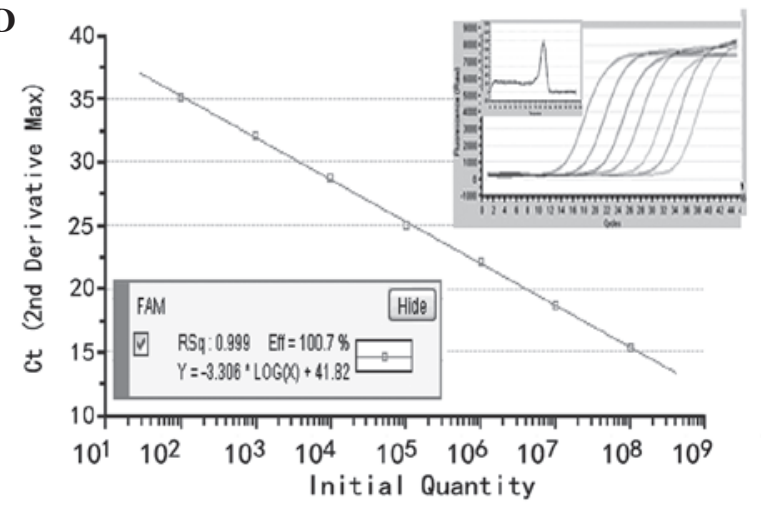

$\mathbf{E}$

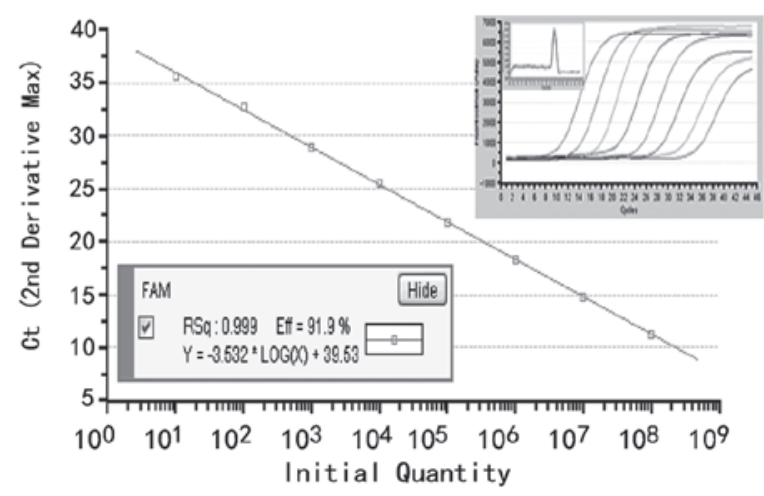

Figure 1. Standard curves were created with 10-fold serially diluted plasmid DNA containing (A) miR-21, (B) miR-31, (C) miR-96, (D) miR-135b or (E) U6 by SYBR-Green I real-time PCR. The Ct values obtained from the real-time PCR assays were plotted against the initial plasmid DNA copy number. The curves demonstrated a wide linear range: $10^{1}-10^{8}$ copies for miR-21, $10^{2}-10^{9}$ copies for miR-31, $10^{2}-10^{8}$ copies for miR-96 and miR-135b and $10^{1}-10^{8}$ copies for U6 snRNA. Correlation coefficients were >0.996, and the melting-curves of miR-21, miR-31, miR-96, miR-135b and U6 are shown as a single, sharply-narrow peak, indicating that pure, homogeneous qPCR products were produced.

Normalization and data analysis. In the present study, we applied a quantification method using U6 as an internal reference to analyze the expression of the miRNAs. The cycle number at the threshold level of the log-based fluorescence is defined as the $\mathrm{Ct}$ value. According to the principle that the $\mathrm{Ct}$ value is inversely proportional to the logarithm of the initial copy number, the copy number of the target miRNA can be accurately and quantitatively calculated based on the construction of a standard curve. For each sample, the normalized expression of the miRNAs was calculated according to the equation (24): En = Copy (target)/Copy (reference); where En is the normalized expression of the miRNA in either tumor tissue or normal mucosal tissue, copy (target) is the copy number of the target miRNA by comparing it to the corresponding standard curve, and copy (reference) is the copy number of U6 by comparing it with the corresponding standard curve. The fold change of the miRNA expression level in tumor tissue was calculated as: $\mathrm{FC}=\mathrm{E}_{\mathrm{T}} / \mathrm{E}_{\mathrm{N}}$; where $\mathrm{E}_{\mathrm{T}}$ is the normalized expression in the tumor tissue and $\mathrm{E}_{\mathrm{N}}$ is the normalized expression in the normal mucosal tissue.

Statistical analysis. An independent sample t-test was used to compare the differential expression between the tumor tissues and the normal mucosal tissues. Statistical differences between 
A

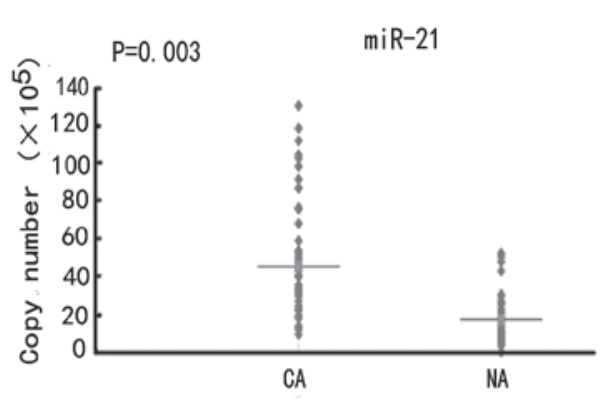

C

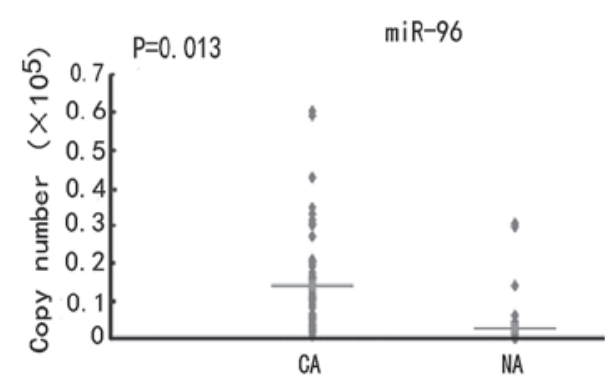

B
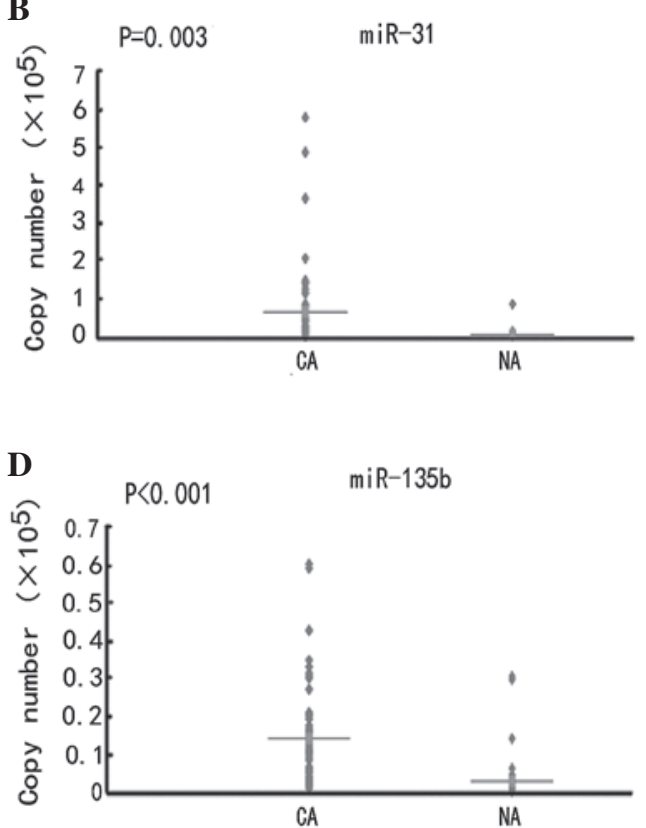

Figure 2. Scatter graphs showing the copy number of miRNAs in CA and NA. (A) miR-21, (B) miR-31, (C) miR-96 and (D) miR-135b were all significantly $(\mathrm{P}<0.05)$ upregulated when compared to normal mucosal tissues. CA, cancer tissues; NA, normal tissues.

the clinicopathological parameters and the miRNA levels were evaluated using non-parametric tests. The Mann-Whitney U test was used between 2 groups and the Kruskall-Wallis test was used between 3 or more groups. $\mathrm{P}<0.05$ was considered to indicate a statistically significant difference. All calculations were performed using SPSS version 16.0 software.

\section{Results}

Sensitivity and specificity of detecting miRNAs. Standard curves were created by plotting the input copy number of a standard plasmid DNA, which contained 62-65 bp of the miR-21, miR-31, miR-96, miR-135b or U6 and the Ct value. Each of the plasmid DNAs were confirmed using sequencing to guarantee the authenticity of the PCR products. Using serial 10-fold dilutions of the plasmid standard, a wide linear range of $10^{1}-10^{8}$ copies for miR-21, $10^{2}-10^{9}$ copies for miR-31, $10^{2}-10^{8}$ copies for miR-96 and miR-135b, and $10^{1}-10^{8}$ copies for U6 snRNA, was detected. Dissociation curves of each miRNA are shown as single, sharply-defined narrow peaks, indicating that specific, homogeneous PCR products were produced (Fig. 1).

Expression of miRNAs in the tumor and corresponding normal tissues. The expression levels of all analyzed miRNAs were significantly different between the tumor and normal adjacent tissues. Expression levels of miR-21, miR-31, miR-96 and miR-135b were upregulated in CRC tissues by $1.5,33.5$, 2.2 and 17.9 times, respectively, compared to the normal tissues $(\mathrm{P}<0.05)$ (Table I; Fig. 2). The mean fold changes of the miRNAs analyzed in the colorectal tumor samples and their possible correlation with cancer and putative targets were detected (Table I).

Among the 52 CRC tissues, 35 (67.3\%), 47 (90.4\%), $42(80.8 \%)$ and $48(92.3 \%)$ tumors demonstrated overexpres- sion of miR-21, miR-31, miR-96 and miR-135b, respectively. The average levels of miRNA expression in the tumor and normal tissues are shown in Table II. We also examined the frequency of the combined upregulated expression of the four miRNAs and revealed that there were 31 cases $(31 / 52,59.6 \%)$ where all four miRNAs were upregulated and 49 cases $(49 / 52$, $94.2 \%$ ) where at least one of the miRNAs was upregulated (data not shown).

Correlation between miRNAs and clinical parameters. miR-96 and miR-135b were found to be associated with liver metastasis ( $\mathrm{p}=0.006$ and $\mathrm{p}=0.013$, respectively) (Table II and Fig. 3). miR-21 expression is associated with lymph node metastasis $(\mathrm{P}=0.032)$ and clinical stage $(\mathrm{P}=0.048)$. However, no correlations were observed between miR-31 and gender, localization, lymph node metastasis, differentiation or clinical stage. Notably, the miR-31 expression was higher in stage III compared to II, but decreased in stage IV. A possible explanation for this phenomenon may involve the stress response. Although the mean expression levels of miR-96 increased progressively with the disease stage, a statistical significance was not achieved.

\section{Discussion}

miRNAs are emerging as major contributors in normal and diseased cell processes and have been demonstrated to be involved in oncogenesis, disease progression, invasion and metastasis (14). To date, a number of different approaches to quantify miRNAs have been described, including northern blotting $(25,26)$, microarrays $(15)$, bead-based hybridization (27), modified invader assays (28) and real-time PCR. Among these approaches, real-time PCR is a more quantitative and sensitive method when compared to other high-throughput assays. 
A

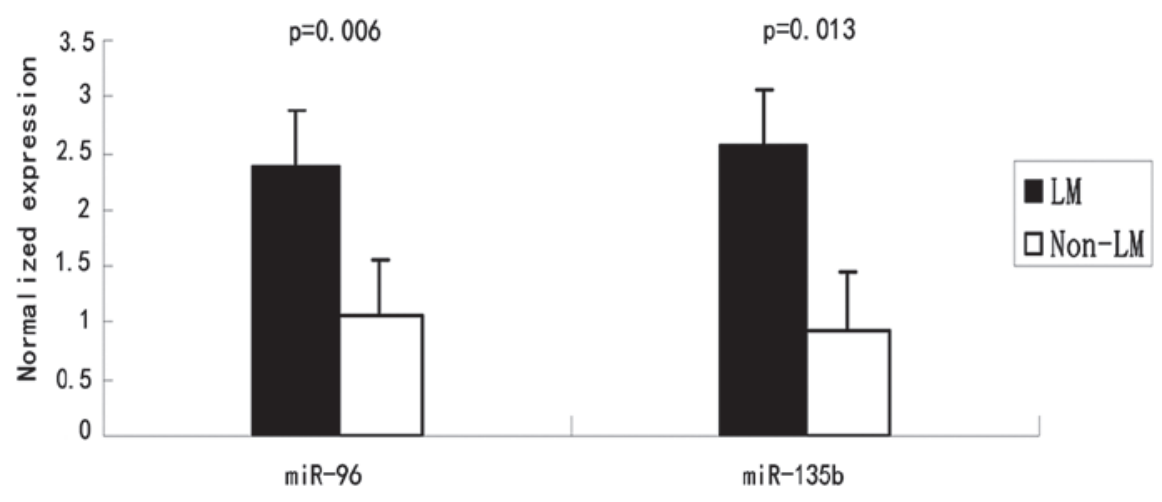

B

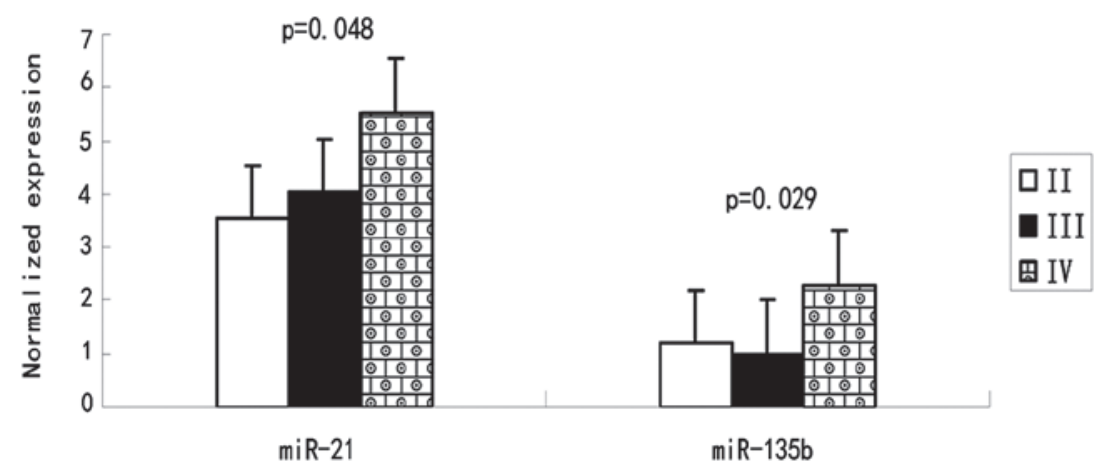

Figure 3. Correlation between the normalized expression levels of miRNAs and clinicopathological features. (A) Normalized expression levels of miR-96 and miR-135b between LM tissues and non-LM tissues. (B) Normalized expression levels of miR-21 and miR-135b among clinical stages II, III and IV. LM, liver metastasis; Non-LM, non-liver metastasis.

Real-time PCR based on TaqMan probes and SYBR-Green I is the most commonly used method for miRNA detection. TaqMan probes, which are designed to hybridize to an internal stretch of the amplicon, are more specific. However, with this method, one miRNA must match a single and unique probe, thereby increasing the overall cost. In contrast, real-time RT-PCR that uses SYBR-Green I is a more cost-effective method. It should be considered that SYBR-Green I is not able to discriminate between different PCR products. Additionally, it binds to all dsDNA, including non-specific products, such as primer dimers. Thus, melting point analysis must be performed to monitor the homogeneity of qPCR products when using the SYBR-Green I method.

Although altered expression levels of numerous miRNAs have been identified in human cancers, limited information is available regarding their physiological and pathological roles. In the present study, we established a specific and sensitive SYBR-Green I real-time RT-PCR method to detect miRNA expression. The melting-curves of miR-21, miR-31, miR-96, miR-135b and U6 were each shown as a single, sharply-defined melting curve with a narrow peak, indicating that pure, homogeneous qPCR products were produced (Fig. 1).

In our study, we analyzed 52 cases of CRC tissues and corresponding normal mucosal tissue, including 7 cases that were liver metastasis-positive and 45 cases that were liver metastasis-negative, in order to identify the expression levels of miR-21, miR-31, miR-96 and miR-135b. The expression levels of the four miRNAs were significantly higher in the tumor tissues than in the matched normal musocal tissues $(\mathrm{P}<0.05)$, which supports previous studies $(10,15,19,29)$. Furthermore, we identified that the expression of miR-21 was not only associated with lymph node metastasis, but also with the clinical stage; this also corresponds with a previous study (10). Bandrés et al (15) examined 12 matched pairs of tumoral and non-tumoral adjacent tissues and revealed that miR-31 was correlated with stage of CRC ( $\mathrm{P}=0.028)$. However, we did not observe this correlation, nor did we find any other significant link between miR-31 and the clinical features examined. Slaby et al (10) examined 29 primary colorectal carcinomas and 6 non-tumoral adjacent tissue specimens using real-time PCR and also found that miR-21 and miR-31 were upregulated in CRC patients. In our study, miR-21 was correlated with the clinical stage $(\mathrm{P}=0.032)$, but miR-31 did not significantly change in the different clinical stages. This discrepancy may partly be due to differences in the specimens analyzed.

We also found that miR-96 and miR-135b were upregulated in the CRC samples and were correlated with liver metastasis ( $\mathrm{P}=0.006$ and $\mathrm{P}=0.013$, respectively). To the best of our knowledge, this is the first study to describe the relationship between miR-96 and miR-135b in CRC patients with liver metastasis. We found no correlation between miR-96 and miR-135b and other clinicopathological characteristics such as gender, localization, lymph node metastasis, differentiation or clinical stage (Table II).

Further studies are required to determine the interactions of miR-21, miR-31, miR-96 and miR-135b with their potential targets. The genes targeted by miR-21 have been under extensive study. The PTEN tumor suppressor gene was first selected 
as a potential miR-21 target in hepatocellular cancer based on its well-characterized role in tumor biology (7). Following this, TPM and PDCD4 were confirmed as functionally significant targets for miR-21 in breast cancer $(16,30)$. It was demonstrated that miR-21 promoted cell migration, invasion and metastasis by downregulating PDCD4 gene expression. Studies on other cancer types have also confirmed that miRNAs are able to promote metastasis and have confirmed their role in tumor migration and invasion (31). miR-31 is able to directly target the homeobox gene SATB2, which is responsible for chromatin remodeling and regulation of gene expression in cancer-associated fibroblasts (24).

miR-135b regulates APC expression and the Wnt signaling pathway, suggesting its contribution to CRC pathogenesis (19). miR-96 was upregulated in colorectal, bladder and prostate cancer, but downregulated in pancreatic cancer $(6,13,15,26)$, where it targets the K-ras oncogene and acts as a tumor suppressor gene (26). From these results it is evident that miRNAs may function as oncogenes or tumor suppressors depending on the tissue type or target gene expression.

In conclusion, we have established a sensitive and specific assay to detect miRNA expression. miR-21, miR-31, miR-96 and miR-135b were upregulated in tumor tissues compared to adjacent normal mucosal tissues. These miRNAs may have roles as oncogenes during the development of CRC. miR-135b, in particular, may be correlated with malignancy and the process of liver metastasis in CRC.

\section{Acknowledgements}

This study was supported by the Key International Science and Technology Cooperation Projects of China (No. 2006DFB31410) and the National Natural Science Foundation of China (No. 81171028). We are grateful to the members of the Institute of Geriatrics of the Ministry of Health for their advice and assistance.

\section{References}

1. Jemal A, Siegel R, Xu J and Ward E: Cancer statistics. CA Cancer J Clin 60: 277-300, 2010.

2. Mandel JS, Bond JH, Church TR, et al: Reducing mortality from colorectal cancer by screening for fecal occult blood. Minnesota Colon Cancer Control Study. N Engl J Med 328: 1365-1371, 1993.

3. Jeon CH, Lee HI, Shin IH and Park JW: Genetic alterations of APC, K-ras, p53, MSI, and MAGE in Korean colorectal cancer patients. Int J Colorectal Dis 23: 29-35, 2008.

4. Slack FJ and Weidhaas JB: MicroRNA in cancer prognosis. N Engl J Med 359: 2720-2722, 2008.

5. Huang GL, Zhang XH, Guo GL, et al: Clinical significance of miR-21 expression in breast cancer: SYBR-Green I-based real-time RT-PCR study of invasive ductal carcinoma. Oncol Rep 21: 673-679, 2009.

6. Schaefer A, Jung M, Mollenkopf HJ, et al: Diagnostic and prognostic implications of microRNA profiling in prostate carcinoma. Int J Cancer 126: 1166-1176, 2010.

7. Meng F, Henson R, Wehbe-Janek H, et al: MicroRNA-21 regulates expression of the PTEN tumor suppressor gene in human hepatocellular cancer. Gastroenterology 133: 647-658, 2007.

8. Filipowicz W, Bhattacharyya SN and Sonenberg N: Mechanisms of post-transcriptional regulation by microRNAs: are the answers in sight? Nat Rev Genet 9: 102-114, 2008
9. Bartel DP: MicroRNAs: genomics, biogenesis, mechanism, and function. Cell 116: 281-297, 2004.

10. Slaby O, Svoboda M, Fabian P, et al: Altered expression of miR-21, miR-31, miR-143 and miR-145 is related to clinicopathologic features of colorectal cancer. Oncology 72: 397-402, 2007.

11. Liu CJ, Kao SY, Tu HF, et al: Increase of microRNA miR-31 level in plasma could be a potential marker of oral cancer. Oral Dis 16: 360-364, 2010

12. Borkhardt A, Fuchs U and Tuschl T: MicroRNA in chronic lymphocytic leukemia. N Engl J Med 354: 524-525, 2006.

13. Han Y, Chen J, Zhao X, et al: MicroRNA expression signatures of bladder cancer revealed by deep sequencing. PLoS One 6: e18286, 2011.

14. Aslam MI, Taylor K, Pringle JH and Jameson JS: MicroRNAs are novel biomarkers of colorectal cancer. Br J Surg 96: 702-710, 2009.

15. Bandrés E, Cubedo E, Agirre X, et al: Identification by Real-time PCR of 13 mature microRNAs differentially expressed in colorectal cancer and non-tumoral tissues. Mol Cancer 5: 29, 2006.

16. Zhu S, Si ML, Wu H and Mo YY: MicroRNA-21 targets the tumor suppressor gene tropomyosin 1 (TPM1). J Biol Chem 282: 14328-14336, 2007.

17. Frankel LB, Christoffersen NR, Jacobsen A, et al: Programmed cell death 4 (PDCD4) is an important functional target of the microRNA miR-21 in breast cancer cells. J Biol Chem 283: 1026-1033, 2008

18. Cottonham CL, Kaneko S and Xu L: miR-21 and miR-31 converge on TIAM1 to regulate migration and invasion of colon carcinoma cells. J Biol Chem 285: 35293-35302, 2010.

19. Nagel R, le Sage C, Diosdado B, et al: Regulation of the adenomatous polyposis coli gene by the miR-135 family in colorectal cancer. Cancer Res 68: 5795-5802, 2008.

20. Dhanasekaran S, Doherty TM and Kenneth J: Comparison of different standards for real-time PCR-based absolute quantification. J Immunol Methods 354: 34-39, 2010.

21. Whelan JA, Russell NB and Whelan MA: A method for the absolute quantification of cDNA using real-time PCR. J Immunol Methods 278: 261-269, 2003

22. Lee C, Kim J, Shin SG and Hwang S: Absolute and relative QPCR quantification of plasmid copy number in Escherichia coli. J Biotechnol 123: 273-280, 2006.

23. Chen C, Ridzon DA, Broomer AJ, et al: Real-time quantification of microRNAs by stem-loop RT-PCR. Nucleic Acids Res 33: e179, 2005.

24. Aprelikova O, Yu X, Palla J, et al: The role of miR-31 and its target gene SATB2 in cancer-associated fibroblasts. Cell Cycle 9: 4387-4398, 2010

25. Michael MZ, SM OC, van Holst Pellekaan NG, et al: Reduced accumulation of specific microRNAs in colorectal neoplasia. Mol Cancer Res 1: 882-891, 2003.

26. Yu S, Lu Z, Liu C, et al: miRNA-96 suppresses KRAS and functions as a tumor suppressor gene in pancreatic cancer. Cancer Res 70: 6015-6025, 2010.

27. Lu J, Getz G, Miska EA, et al: MicroRNA expression profiles classify human cancers. Nature 435: 834-838, 2005.

28. Allawi HT, Dahlberg JE, Olson S, et al: Quantitation of microRNAs using a modified Invader assay. RNA 10: 1153-1161, 2004.

29. Wang CJ, Zhou ZG, Wang L, et al: Clinicopathological significance of microRNA-31, -143 and -145 expression in colorectal cancer. Dis Markers 26: 27-34, 2009.

30. Lu Z, Liu M, Stribinskis V, et al: MicroRNA-21 promotes cell transformation by targeting the programmed cell death 4 gene. Oncogene 27: 4373-4379, 2008.

31. Huang Q, Gumireddy K, Schrier M, et al: The microRNAs miR-373 and miR-520c promote tumour invasion and metastasis. Nat Cell Biol 10: 202-210, 2008.

32. Lee EJ, Gusev Y, Jiang J, et al: Expression profiling identifies microRNA signature in pancreatic cancer. Int J Cancer 120: 1046-1054, 2007.

33. Tong AW, Fulgham P, Jay C, et al: MicroRNA profile analysis of human prostate cancers. Cancer Gene Ther 16: 206-216, 2009. 